\title{
Distribución de grupos filogenéticos, factores de virulencia y susceptibilidad antimicrobiana en cepas de Escherichia coli uropatógena
}

\author{
Distribution of phylogenetic groups, virulence factors and antimicrobial susceptibility \\ in strains of uropathogenic Escherichia coli
}

\author{
Ysheth Millán², María Araque² y Ana Ramírez ${ }^{1,2}$
}



Recibido (versión final): 18 de diciembre de 2019 / Aceptado: 31 de enero de 2020

\section{Resumen}

Introducción: La diferencia entre los aislados patógenos y comensales de Escherichia coli se fundamenta en sus antecedentes filogenéticos. En Venezuela son escasos los estudios que describen el potencial patogénico de los grupos filogenéticos en E. coli. Objetivo: Relacionar la susceptibilidad antimicrobiana, distribución de los grupos filogenéticos y genes de virulencia en cepas de $E$. coli uropatógena (ECUP) aisladas de pacientes con infección del tracto urinario. Materiales y Métodos: Se estudiaron 17 cepas de ECUP, aisladas de pacientes adultos hospitalizados en dos instituciones de salud. La susceptibilidad frente a ocho antimicrobianos se determinó por el método de microdilución en caldo (MDC). Las $\beta$-lactamasas de espectro extendido (BLEE) y carbapenemasas fueron detectadas fenotípicamente. Los grupos filogenéticos y la detección de los genes de virulencia se determinaron por reacción de polimerasa en cadena. Resultados: Todas las cepas sintetizaban BLEE y de éstas, $41 \%$ se asoció a la producción de una carbapenemasa (KPC o MBL). El filogrupo B2 (41\%) fue predominante. Los genes de virulencia más frecuentes fueron fim $H$ y fyuA con $82 \%$ cada uno. Sólo un aislado clasificado en el filogrupo $\mathrm{F}$ fue positivo al conjunto de seis genes estudiados. Discusión: La diversidad de asociaciones entre genes de virulencia y perfiles de resistencia, en las cepas ECUP evolucionan continuamente. Además, su distribución en los distintos grupos filogenéticos depende en gran medida de las características clínico epidemiológicas de los grupos de estudios.

Palabras clave: Escherichia coli uropatógena; grupos filogenéticos; genes de virulencia; $\beta$-lactamasas.

\begin{abstract}
Background: The difference between the pathogenic isolates and commensals of Escherichia coli is based on their phylogenetic antecedents. In Venezuela there are few studies that describe the pathogenic potential of phylogenetic groups in E. coli. Aims: Relate antimicrobial susceptibility, distribution of phylogenetic groups and virulence genes in strains of uropathogenic $E$. coli (ECUP) isolated from patients with UTI. Methods: We studied 17 ECUP strains, isolated from adult patients hospitalized in two health institutions. The susceptibility to 8 antibiotics was determined by the broth microdilution (MDC) method. Extended spectrum $\beta$-lactamases (ESBL) and carbapenemases were phenotypically detected. The phylogenetic groups and the detection of the virulence genes were determined by PCR. Results: All strains synthesized ESBL and of these, $41 \%$ were associated with the production of a carbapenemases (KPC or MBL). The phylogroup B2 (41\%) was the most predominant. The most frequent virulence genes were fim $H$ and $f y u A$ with $82 \%$ each. Only one strain from group F was positive to the 6 genes studied. Discussion: The diversity of associations between virulence genes and resistance profiles in the ECUP are evolving continuously, their distribution in the different phylogenetic groups depends to a large extent on the clinical epidemiological characteristics of the study groups.

Keywords: Uropathogenic Escherichia coli; phylogenetic groups, virulence genes, $\beta$-lactamases.
\end{abstract}




\section{Introducción}

L as infecciones del tracto urinario (ITU) afectan a 150 millones de personas cada año en todo el mundo, siendo Escherichia coli uropatógena (ECUP) el agente etiológico responsable de aproximadamente 50\% de las ITU asociadas a los cuidados de salud (ITU-ACS) ${ }^{1,2}$ y de 70 a $95 \%$ en la comunidad ${ }^{3}$.Venezuela no escapa de esta realidad mundial y, aunque los estudios publicados sobre la distribución de E. coli como agente causal de las ITU son escasos en nuestro país, autores como López y cols., en niños, Capozzi y cols., y Quijada y cols., en adultos, reportan frecuencias de 23,2\% (44/189), 53,9\% (7/13) y $20 \%(8 / 40)$, respectivamente ${ }^{4-6}$.

La capacidad de ECUP de producir ITU depende de una compleja interacción entre las condiciones del hospedero y los diferentes factores de virulencia (FV) que posea la bacteria ${ }^{7}$, siendo las fimbrias: tipo 1 (codificado por el operon fim), P (codificada por operon pap), F1C (foc $G$ ), $\mathrm{S}(S f a)$ y las adhesinas no fimbriales $\operatorname{Dr}(A f a / d r B C)$ y afa $(a f a B / C)$, las que intervienen en las etapas de colonización y adherencia, cuya presencia o ausencia en $E$. coli puede variar entre los distintos tipos de ITU (bacteriuria, cistitis o pielonefritis). Otros FV como la cápsula ( $k p s M T)$ son importantes en la evasión de la respuesta inmune y en los

\begin{tabular}{lccl}
\multicolumn{4}{l}{ Tabla 1. Datos clínicos y epidemiológicos de los pacientes con ITU incluidos en el estudio } \\
\hline Aislados clínicos & Sexo & $\begin{array}{c}\text { Edad } \\
\text { (años) }\end{array}$ & Patología o estado fisiológico asociado \\
\hline LMM-1147 & F & 73 & ITU-AC \\
\hline LMM-1195 & F & 43 & ITU-AC \\
\hline LMM-15131.1 & F & 53 & ITU-AC \\
\hline LMM-15131.2 & F & 42 & ITU-AC \\
\hline LMM-1194.3 & M & 34 & ITU-AC \\
\hline LMM-1199 & F & 68 & ITU-AC \\
\hline LMM-15494.2 & M & 70 & ITU-AC \\
\hline A-316 & M & 68 & Cistocele con ITU \\
\hline A-099 & F & 79 & Atrofia renal con ITU \\
\hline A-170 & M & 59 & Atrofia renal con ITU \\
\hline A-717 & F & 50 & Cistocele con ITU \\
\hline A-719 & M & 28 & ITU-AC \\
\hline A-967 & F & 17 & Embarazo con ITU \\
\hline A-038 & F & 51 & Diabetes mellitus con ITU \\
\hline A-726 & M & 74 & ITU-AC \\
\hline A-767 & F & 51 & Lupus eritematoso con ITU \\
\hline A-178 & M & 75 & ITU-AC \\
\hline
\end{tabular}

ITU-AC: Infección urinaria asociada a catéter vesical. $M=$ masculino; $F=$ femenino. cuadros invasores. Además, los sideróforos intervienen en la adquisición de nutrientes mientras que las toxinas $\alpha$-hemolisina $(h l y A)$, el factor citotóxico necrosante (cnfl) y la toxina auto-transportadora Sat (Sat) son importantes en el tipo de daño causado al tejido renal, por lo que ayudan a definir el cuadro clínico producido por las ECUP en el tracto urinario ${ }^{7,8}$.

Por otra parte, las cepas de E. coli son genéticamente diversas $^{9}$, de tal manera que se han descrito 8 grupos filogenéticos (A, B1, B2, C, D, E, F y clado 1), utilizando datos obtenidos mediante la tipificación de secuencias multilocus (MLST) ${ }^{10}$. Sin embargo, Clermont y cols., en 2013 describieron y validaron una nueva metodología para realizar dicha clasificación, basándose en el genotipo obtenido mediante reacción de polimerasa en cadena (RPC) múltiple que detecta los genes $\operatorname{arp} A, \operatorname{chu} A$, yjaA y el fragmento génicoTspE4.C2. Estos autores demostraron que su metodología asigna correctamente 95 a $100 \%$ de las cepas al compararla con la técnica MLST ${ }^{11}$.

En general, se ha reportado que las cepas del grupo B2 expresan una mayor cantidad de FV y son las más frecuentes en casos de cistitis y bacteriuria asintomática complicada $^{12}$; además, están asociadas con mayor frecuencia con la producción de $\beta$-lactamasas ${ }^{13}$.

En Venezuela son escasos los estudios que describen las bases genéticas que sustentan el potencial patogénico de los grupos filogenéticos de ECUP ${ }^{12,14,15}$. Por tal motivo, en este trabajo se relacionó la susceptibilidad antimicrobiana, distribución de los grupos filogenéticos y genes de virulencia en cepas de ECUP aisladas de pacientes adultos con ITU.

\section{Materiales y Métodos}

Este estudio fue de tipo experimental, exploratorio y descriptivo.

\section{Cepas bacterianas}

Se estudiaron 17 cepas de ECUP, pertenecientes a la colección del Laboratorio de Microbiología Molecular de la Facultad de Farmacia y Bioanálisis de la Universidad de Los Andes, Mérida-Venezuela (LMMFFB-ULA), las que fueron obtenidas de muestras de orina provenientes de pacientes adultos hospitalizados, 10 cepas durante el período marzo de 2011 a julio de 2013 y 7 durante el período enero a julio de 2015. Los criterios de inclusión fueron: cepas de E. coli donadas al LMMFFB-ULA, con sospecha de BLEE, aisladas de pacientes adultos con ITU; se excluyeron cepas de E. coli que no cumplían con los criterios antes mencionados. En la Tabla 1 se muestran las características clínicas y epidemiológicas de los pacientes a partir de quienes se obtuvieron las cepas de ECUP incluidas en este estudio. 
por Clermont y cols. ${ }^{11}$ (Tabla suplementaria 1). Los resultados fueron interpretados utilizando el esquema de Clermont y cols. ${ }^{11}$. Para estos ensayos se utilizaron como cepas control: E. coli 09-ULA (chuA+, yjaA+ y TspE4. $\mathrm{C} 2+)$, E. coli $A 038-\mathrm{ULA}(\operatorname{arp} A+\mathrm{y} \operatorname{arp} A \mathrm{gE}+)$ y $E$. coli $\operatorname{SC} 20-\mathrm{ULA}(\operatorname{trp} A+)$.

\section{Detección de genes de virulencia}

Se estudiaron seis genes de virulencia, incluyendo: cápsula polisacárida específica del grupo II ( $k p s M T I I)$, adhesina de la fimbria tipo 1 (fimH), fimbria $\mathrm{P}$ ( $p a-$ $p A H)$, marcador de isla de patogenicidadI ${ }_{\mathrm{CFT} 073}$ (PAI), yersiniabactina (sideróforo $f y u A$ ) y proteína específica uropatógena (usp). Los genes kpsMTII, fimH y PAI fueron detectados mediante una RPC múltiple y el resto ( $p a p A H$, fyuAy usp) por RPC simple, utilizando los iniciadores y condiciones de amplificación previamente descritos ${ }^{12}$ (Tabla suplementaria 1). Las cepas control utilizadas en estos ensayos fueron E. coli LMM/E02-ULA ( $f i m H+$, fyuA+, kpsMTII + y PAI +), E. coli LMM/Sc03-ULA $($ pap $A H+)$ y E. coli LMM/E02-ULA $(u s p+)$.

\section{Resultados}

Un total de 17 aislados clínicos de ECUP fueron evaluados por el método de MDC frente a ocho antimicrobianos. En todas las cepas se confirmó fenotípicamente la producción de BLEE y de éstas 41\% (7/17) se asoció a la síntesis de una carbapenemasa (KPC o MBL). Sin embargo, tres $(18 \%)$ de las cepas resistentes al imipenem no resultaron positivas en la prueba fenotípica para carbapenemasas. También se observó resistencia a ciprofloxacina en $100 \%$ de los aislados.

Por otra parte, al analizar la distribución de las cepas con base en los grupos filogenéticos, se determinó que el filogrupo B2 (41\%) fue el predominante, seguido de los grupos A y E con 23,5\% (4/17) cada uno y sólo 12\% (2/17) correspondió al filogrupo F (Tabla suplementaria 2). Todas las cepas del grupo A fueron productoras de BLEE y carbapenemasas, mientras que las clasificadas dentro del filogrupo F sólo sintetizaban BLEE y las del grupo B2 mostraron un perfil de resistencia más heterogéneo (Tabla suplementaria 2).

En relación a la presencia de genes de virulencia, los más frecuentes fueron fimHy fyuA con $82 \%(14 / 17)$ cada uno, seguidos de usp (53\%), KpsMTII (47\%) PAI (41\%) y papAH (18\%). Sólo un aislado clasificado en el filogrupo F fue positivo al conjunto de seis genes estudiados (Tabla suplementaria 2), mientras que dos cepas del grupo A sólo presentaron el gen fim $H$. La mayoría de los aislados pertenecientes a los filogrupos B2 y E presentaron entre tres y cinco genes de virulencia. 


\section{Discusión}

Las ECUP incluidas en este estudio presentaron un alto porcentaje (mayor al $65 \%$ ) de resistencia frente a los ocho antimicrobianos ensayados, resultado que es consistente con lo reportado por varios autores ${ }^{19,20}$, sobre la multi-resistencia de $E$. coli frente a los antimicrobianos.

Por otra parte, $65 \%$ de los aislados clínicos fueron resistente a carbapenemos y en $41 \%$ de éstos, el mecanismo de resistencia implicado fue la síntesis de carbapenemasas (KPC y MBL), lo que revela un cambio importante en el patrón de susceptibilidad frente a este grupo de antimicrobianos al compararlo con los resultados reportados en el 2014 por Abreu y cols. ${ }^{21}$, quienes señalaron que $100 \%$ de las cepas ECUP de origen hospitalario en MéridaVenezuela, eran sensibles a carbapenemos. Es probable que al utilizar este grupo de antimicrobianos como opción terapéutica en infecciones causadas por bacterias productoras BLEE, se ejerciera presión selectiva y las bacterias rápidamente adquieran mecanismos para evadir la acción de estos antimicrobianos ${ }^{22}$.

De igual forma, el elevado porcentaje de resistencia $(100 \%)$ frente a ciprofloxacina y la asociación de dicha resistencia con la síntesis de BLEE, se correlaciona con lo referido por Montañez-Valverde y cols. ${ }^{23}$, en cepas ECUP en Perú. Este hallazgo puede indicar la posible circulación de elementos genéticos que codifiquen información sobre mecanismos de resistencia a varias familias de antimicrobianos, aspecto que debe ser confirmado en estudios posteriores.

Con respecto a la distribución filogenética en las cepas de E. coli analizadas se observó, al igual que otros autores ${ }^{1,24}$ en Alemania (B2: 49\%) y en Pakistan (B2: 44\%) que el filogrupo B2 fue el más frecuente en cepas ECUP de origen hospitalario, mientras que un estudio realizado en Mérida-Venezuela con cepas ECUP de origen extra-hospitalario, predominó el filogrupo A $(54 \%)^{12}$, evidenciándose que el origen de la cepa (hospitalario o extra-hospitalario) es una característica importante que se debe considerar cuando se establecen patrones particulares de distribución filogenética en cepas de E. coli $i^{25}$.

$\mathrm{Si}$ bien es cierto que la distribución de los FV en las cepas ECUP es heterogénea y que el potencial patogénico de estas cepas es multifactorial ${ }^{1,12,26}$, nuestros resultados sobre la fimbria tipo 1 ( $f m H)(82 \%)$ y el sideróforo yer- siniabactina $(f y u A)(82 \%)$ coinciden con los reportados por otros autores en Alemania ${ }^{25}$, Mongolia ${ }^{26}$, China ${ }^{27}$ y Venezuela ${ }^{12}$, apoyando así, lo referido por diversos autores sobre la importancia de los genes fyu $\mathrm{A}$ y fim $\mathrm{H}$ como indicadores claves de virulencia en cepas ECUP'2.

El predominio de fyuA en ECUP puede estar relacionado con la alta afinidad por el hierro y la mayor estabilidad que tiene yersiniabactina con respecto a otros sideróforos ${ }^{28}$, aspecto importante para la captación y acumulación de hierro, sobre todo en el tracto urinario que es un ambiente tan limitado de este nutriente ${ }^{27}$. De igual forma, la fimbria tipo 1 ( $\mathrm{fim} H$ ) prevalece en cepas ECUP debido a que sus receptores específicos se encuentran en el uroepitelio favoreciendo la entrada al tracto urinario; además, promueve la formación de biopelículas facilitando la colonización de catéteres vesicales ${ }^{8}$, factor predisponente más frecuente (59\%) en este estudio.

En relación al número de genes de virulencia, se determinó que la mayoría (71\%) de las cepas ECUP analizadas contenían tres o más de los genes investigados. Al compararlo con lo reportado por Millán y cols. ${ }^{12}$, se observa una importante diferencia en la carga genética, ya que estos autores reportan que $61 \%$ de las cepas contenían dos o menos genes de virulencia. Esta característica, aunado a los mecanismos de resistencia detectados, revela el alto grado de virulencia de las ECUP analizadas, pudiendo junto a los factores inherentes al hospedero conllevar a que el cuadro clínico sea más grave en este grupo de pacientes?

A pesar que una limitación del estudio fue el número reducido de cepas analizadas, estos hallazgos, representan un aporte importante en el ámbito científico de nuestra región, ya que permiten conocer cambios en la susceptibilidad antimicrobiana, así como, la carga genética que describe la potencialidad patogénica relacionada a los grupos filogenéticos en cepas ECUP que circulan en las dos instituciones de salud involucradas en el estudio.

Los hallazgos obtenidos en este trabajo permiten concluir que la distribución de las ECUP en los distintos grupos filogenéticos depende en gran medida de las características clínico epidemiológicas de los grupos de estudios y que es evidente que la diversidad de asociaciones entre genes de virulencia, perfiles de resistencia, síntesis de BLEE y carbapenemasas en las cepas ECUP evolucionan continuamente, planteando un reto importante en el tratamiento de las ITU. 


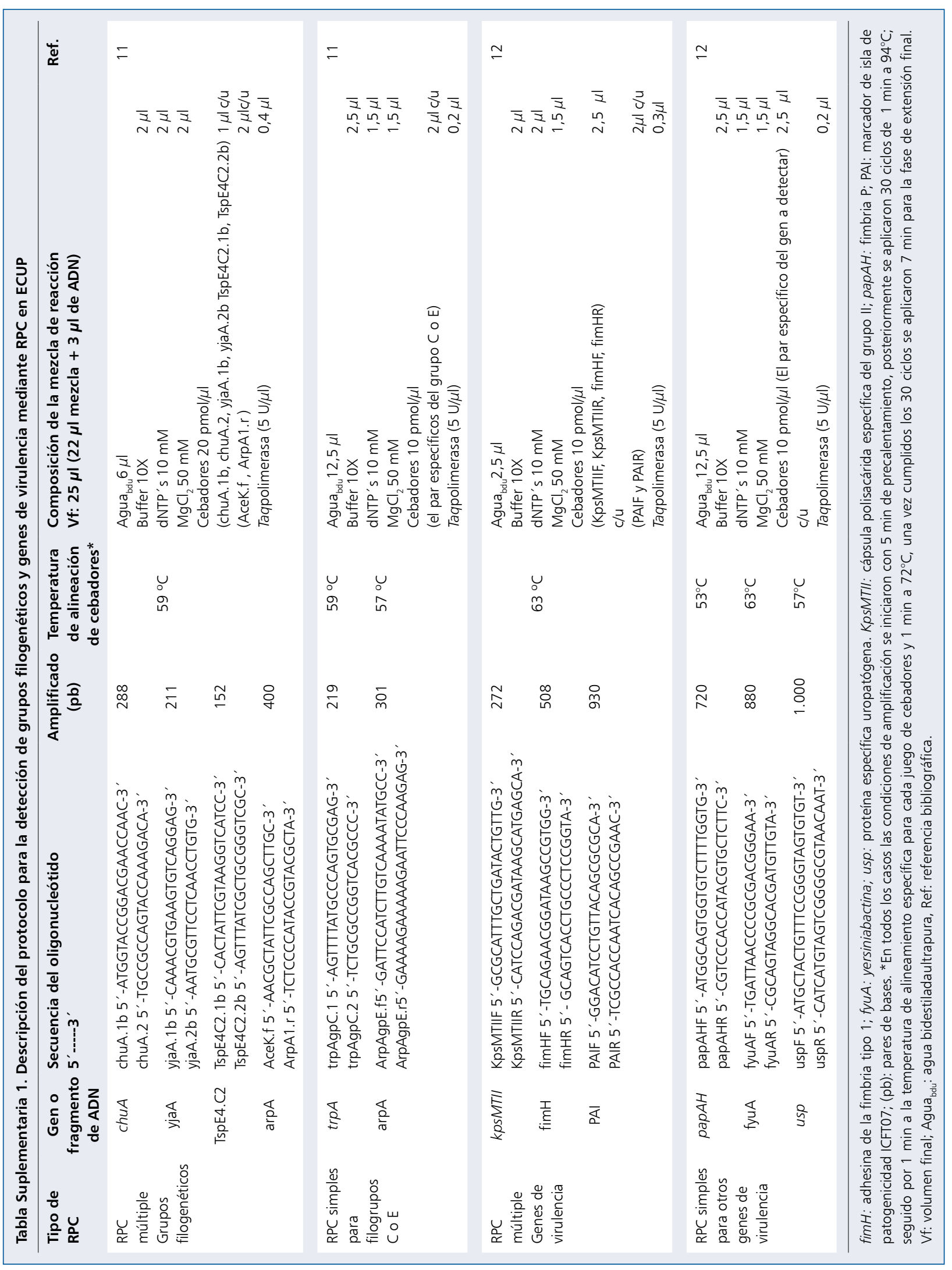




\begin{tabular}{|c|c|c|c|c|}
\hline Código de cepa & Tipo de $\beta$-lactamasa* & $\begin{array}{l}\text { Perfil de resistencia } \\
\text { Resistencia asociada a otros antimicrobianos** }\end{array}$ & Grupo filogenético & Genes de virulencia \\
\hline \multicolumn{5}{|l|}{ IAHULA } \\
\hline LMM-1147 & $\mathrm{BLEE}+\mathrm{MBL}$ & CIP, AK & A & $\mathrm{fimH}$ \\
\hline LMM-1195 & $\mathrm{BLEE}+\mathrm{MBL}$ & CIP, AK & A & $\mathrm{fimH}$ \\
\hline LMM-15131.1 & $\mathrm{BLEE}+\mathrm{KPC}$ & CIP, AK & A & fimH, fyuA, usp \\
\hline LMM-15131.2 & $\mathrm{BLEE}+\mathrm{KPC}$ & CIP, AK & A & fimH, usp \\
\hline LMM-1194.3 & $\mathrm{BLEE}+\mathrm{KPC}$ & CIP, AK & B2 & fimH, fyuA, KpsMTII \\
\hline LMM-1199 & BLEE & CIP & $\mathrm{E}$ & fimH, fyuA, usp \\
\hline LMM-15494.2 & BLEE & $\mathrm{CIP}, \mathrm{AK}, \mathrm{IMP}$ & $\mathrm{F}$ & fimH, fyuA, papAH, KpsMTII, PAI \\
\hline \multicolumn{5}{|l|}{ ULE } \\
\hline A-316 & BLEE & CIP, AK, IMP & B2 & fimH, fyuA, KpsMTII, PAI, usp \\
\hline A-099 & BLEE & CIP, AK, IMP & B2 & fimH, fyuA, KpsMTII, \\
\hline$A-170$ & BLEE & CIP, AK & B2 & fyuA, PAI \\
\hline A-717 & $\mathrm{BLEE}+\mathrm{MBL}$ & CIP, AK & B2 & fimH, fyuA, KpsMTII, usp \\
\hline A-719 & $\mathrm{BLEE}+\mathrm{KPC}$ & CIP, AK & B2 & fimH, fyuA, KpsMTII, PAl, usp \\
\hline A-967 & BLEE & CIP & B2 & fimH, fyuA, papAH, KpsMTII, \\
\hline A-038 & BLEE & CIP, AK & $\mathrm{E}$ & fyuA, PAl, usp \\
\hline A-726 & BLEE & CIP, AK & $E$ & fimH, fyuA, usp \\
\hline A-767 & BLEE & $\mathrm{CIP}$ & $E$ & fyuA, PAl \\
\hline A-178 & BLEE & CIP, AK, IMP & $\mathrm{F}$ & fimH, fyuA, papAH, KpsMTII, PAl, usp \\
\hline $\begin{array}{l}\text { *Detección fenotíp } \\
\text { de Los Andes; ULE } \\
\text { nobactámicos, pip } \\
\text { CIP: ciprofloxacina } \\
\text { polisacárida espec }\end{array}$ & $\begin{array}{l}\text { a; **Otros antimicrobianc } \\
\text { Unidad de Larga Estancia } \\
\text { acilina/tazobactam, ampi } \\
\text { AK: amikacina; IMP: imipe } \\
\text { ca del grupo II; papAH: fir }\end{array}$ & $\begin{array}{l}\text { S no incluidos en el perfil de hidrólisis de las } \beta \text {-lactama } \\
\text { Ambulatorio Venezuela; BLEE: } \beta \text {-lactamasas de espec } \\
\text { cilina/sulbactam y amoxicilina/ácido clavulánico; M } \beta \text { L: } \\
\text { nem; fimH: adhesina de la fimbria tipo 1; fyUA: yersin } \\
\text { nbria P; PAl: marcador de isla de patogenicidad ICFT07 }\end{array}$ & $\begin{array}{l}\text { sas detectadas; IAHULA } \\
\text { ro extendido incluye re } \\
\text { netalo } \beta \text {-lactamasas; K } \\
\text { abactina; usp: proteín }\end{array}$ & $\begin{array}{l}\text { Istituto Autónomo Hospital Universitario } \\
\text { tencia a penicilinas, cefalosporinas, mo- } \\
\text { Klebsiella pneumoniae-carbapenemasa; } \\
\text { specífica uropatógena; KpsMTII: cápsula }\end{array}$ \\
\hline
\end{tabular}

\section{Referencias bibliográficas}

1.- Kumar N, Nahid F, Zahra R. Association of virulence factors, phylogenetic groups and antimicrobial resistance markers in Escherichia coli from Badin city, Pakistan. J Chemother 2017; 29 (1): 8-13. DOI: 10.1080/1120009X.2016.1154682.

2.- Flores-Mireles A, Walker J, Caparon M, Hultgren S. Urinary tract infections: epidemiology, mechanisms of infection and treatment options. Nat Rev Microbiol 2015; 13 (5): 269-84. DOI: 10.1038/nrmicro3432.

3.- Vimont S, Boyd A, Bleibtreu A, Bens M, Goujon J M, Garry L, et al. The CTX-M-15producing Escherichia coli clone 025b:H4ST131 has high intestine colonization and urinary tract infection abilities. PLoS ONE 2012; 7 (9): 1-10. DOI: 10.1371/journal. pone. 0046547 .

4.- López D, Olivero R, Wong R, Aurenty L, Smith S, Nexans M, et al. Prevalencia y mortalidad por infecciones del tracto urinario asociadas a la atención de la salud en pediatría. Bol Venez Infectol 2015; 26 (2): 97-103. http://docs.bvsalud.org/ biblioref/2016/07/957/05-lopez-d-97-103.pdf.

5.- Capozzi E, Mobili D, Kornett A, Perdomo M. Etiologic agents of urinary tract infections in older adults from a health center of Carabobo state, Venezuela.Kasmera 2016; 44 (1): 35-43. http://www.scielo.org.ve/pdf/km/v44n1/art06. pdf.

6.- Quijada-Martínez P, Flores-Carrero A, Labrador I, Araque M. Estudio clínico y microbiológico de la infección urinaria asociada a catéter, en los servicios de medicina interna de un hospital universitario venezolano. Rev Peru Med Exp Salud Publica 2017; 34 (1): 52-61.

7.- $\quad$ Miranda-Estrada L, Ruíz-Rosas M, MolinaLópez J, Parra-Rojas I, González-Villalobos E, Castro-Alarcón N. Relación entre factores de virulencia, resistencia a antibióticos y los grupos filogenéticos de Escherichia coli uropatógena en dos localidades de México. Enferm Infecc Microbiol Clin 2017; 35 (7): 426-33. DOI: 10.1016/j.eimc.2016.02.021.

8.- Bien J, Sokolova O, Bozko P. Role of uropathogenic Escherichia coli virulence factors in development of urinary tract infection and kidney damage. Int J Nephrol 2012; 2012 (U): 1-15. DOI: 10.1155/2012/681473.

9.- Clermont O, Bonacorsi S, Bingen E. Rapid and simple determination of the Escherichia coli phylogenetic group. Appl Environ Microbiol 2000; 66 (10): 4555-8.PMID: 11010916.

10.- Chaudhuri R, Henderson I. The evolution of the Escherichia coli phylogeny. Infect Genet Evol 2012; 12 (2): 214-26. DOI: 10.1016/j. meegid.2012.01.005.

11.- Clermont O, Christenson J K, Denamur E, Gordon D M. The Clermont Escherichia coli phylo-typing method revisited: Improvement of specificity of new phylo-groups. Environ Microbiol Rep 2013; 5 (1): 58-65. DOI: 10.1111/1758-2229.12019.

12.- Millán Y, Hernández E, Millán B, Araque M. Distribución de grupos filogenéticos y factores de virulencia en cepas de Escherichia coli uropatógena productora de $\beta$-lactamasa CTX-M-15 aisladas de pacientes de la comunidad en Mérida, Venezuela. Rev Argent Microbiol 2014; 46 (3): 175-81. DOI: 10.1016/ S0325-7541(14)70069-0. 
13.- Tourret J, Denamur E. Population phylogenomics of extraintestinal pathogenic Escherichia coli. Microbiol Spectrum 2016; 4 (1): 1-20. DOI:10.1128/microbiolspec.UTI0010-2012.

14.- Quijada-Martínez P, Flores-Carrero A, Labrador I, Millán Y, Araque M. Microbiological profile and molecular characterization of multidrugresistant gram-negative bacilli producing catheter-associated urinary tract infections in the internal medicine services of a Venezuelan university hospital. Austin J Infect Dis 2017; 4 (1): 2-8. DOI: ajid-v4-id1030

15.- Varela Y, Millán B, Araque M. Diversidad genética de Escherichia coli extra-intestinales productoras de $\beta$-lactamasas TEM, SHV y CTX-M asociada a los cuidados de la salud. Biomédica 2017; 37 (2): 209-217. DOI:10.7705/biomedica.v37i3.3324

16.- Clinical and Laboratory Standards Institute (CLSI). Performance Standards for Antimicrobial Susceptibility Test; 26th informational supplement. Wayne: CLSI; 2018.

17.- Guevara A, De Waard J, Araque M. Detección del gen bla $_{V I M-2}$ en cepas de Pseudomonas aeruginosa productoras de metalo $\beta$-lactamasa aisladas en una unidad de cuidados intensivos en Ciudad Bolívar, Venezuela. Rev Chilena Infectol 2009; 26 (4): 336-41. DOI:10.4067/ S0716-10182009000500005.

18.- Nicola F, Nievas J, Smayevsky J. Evaluación de diversos métodos fenotípicos para la detección de carbapenemasas KPC en Klebsiella pneumoniae. Rev Argent Microbiol
2012; 44 (4): 290-302. DOI: S032575412012000400010.

19.- Arana D, Rubio M, Alós J. Evolution of antibiotic multiresistance in Escherichia coli and Klebsiella pneumoniae isolates from urinary tract infections: A 12-year analysis (2003-2014) Enferm Infecc Microbiol Clin 2017; 35 (5): 293-8. DOI: 10.1016/j. eimc.2016.02.018.

20.- Hernández E, Araque M, Millán Y, Millán B, Vielma S. Prevalencia de $\beta$-lactamasa CTX-M-15 en grupos filogenéticos de Escherichia coli uropatógena aisladas en pacientes de la comunidad en Mérida, Venezuela. Invest Clin 2014; 55 (1): 32-43. DOI: 372937029005.

21.- Abreu S, Varela Y, Millán B, Araque M. Klebsiella pneumoniae y Escherichia coli productoras de beta-lactamasas de espectro extendido, aisladas en pacientes con infección asociada a los cuidados de la salud en un hospital universitario. Enf Inf Microbiol 2014; 34 (3): 92-9. DOI: ei-2014/ei143d.

22.- Ortega A, Oteo J, Aranzamendi-Zaldumbide M, Bartolomé R, Bou G, Cercenado E, et al. Spanish multicenter study of the epidemiology and mechanisms of amoxicillin clavulanate resistance in Escherichia coli. Antimicrob. Agents Chemother 2012; 56 (7): 3576-81. DOI: 10.1128/AAC.06393-11.

23.- Montañez-Valverde R, Montenegro-Idrogo $\mathrm{J}$, Arenas-Significación F, Vásquez-Alva R. Infección urinaria alta comunitaria por Eschericia coli resistente a ciprofloxacino: características asociadas en pacientes de un hospital nacional en Perú. An Fac Med 2015; 76 (4): 385-91. DOI. 10.15381/anales. v76i4.11408.

24.- Toval F, Köhler C-D, Vogel U, Wagenlehner F, Mellmann A, Fruth A, et al. Characterization of Escherichia coli isolates from hospital inpatients or outpatients with urinary tract infection. J Clin Microbiol 2014; 52 (2): $407-$ 18. DOI: $10.1128 / J C M .02069-13$.

25.- Bailey J, Pinyon J. Distribution of human commensal Escherichia coli phylogenetic groups Anantham S, Hall R. J Clin Microbiol 2010; 48 (9): 3455-6. DOI: 10.1128/ JCM.00760-10.

26.- Munkhdelger Y, Gunregjav N, Dorjpurev A, Juniichiro N, Sarantuya J. Detection of virulence genes, phylogenetic group and antibiotic resistance of uropathogenic Escherichia coli in Mongolia. Infect Dev Ctries 2017; 11 (1): 51-7. DOI: 10.3855/ jidc.7903.

27.- Luo Y, Ma Y, Zhao Q, Wang L, Guo L, Ye L, et al. Similarity and divergence of phylogenies, antimicrobial susceptibilities, and virulence factor profiles of Escherichia coli isolates causing recurrent urinary tract infections that persist or result from reinfection. J Clin Microbiol 2012; 50 (12): 4002-7. DOI: 10.1128/JCM.02086-12.

28.- Crosa J, Walsh C. Genetics and assembly line enzymology of siderophore biosynthesis in bacteria. Microbiol Mol Biol Rev 2002; 66 (2): 223. DOI: $10.1128 / \mathrm{mmbr}$.66.2.223-249.2002. 